\title{
Paramagnetic Capture Mode Magnetophoretic Microseparator for Blood Cells
}

\author{
Ki-Ho Han, and A. Bruno Frazier \\ School of Electrical and Computer Engineering, Georgia Institute of Technology \\ Atlanta, GA 30332-0250
}

\begin{abstract}
This paper presents the characterization of a continuous paramagnetic capture (PMC) mode magnetophoretic microseparator for separating white and red blood cells from whole blood by using a high gradient magnetic separation (HGMS) method and microfabrication technology. The PMC microseparator directly separates blood cells based on their native magnetic properties without the use of additives such as magnetic tagging or inducing materials. Experimental results show that the present PMC microseparator can continuously separate out $68.2 \%$ of the red blood cells (RBC's) from whole blood in 5 minutes by applying an external magnetic flux of $0.2 \mathrm{~T}$ using a permanent magnet.
\end{abstract}

\section{INTRODUCTION}

Conventional magnetophoretic macroseparators [1] using HGMS have been used for separating particles from liquids because of characteristics such as a non-hydrolytic separation force, simple structure, and ease of use. In ref. [2], we reported the development of a diamagnetic capture (DMC) mode magnetophoretic microseparator, which separated WBC's, and RBC's. The DMC mode utilized a permanent magnet to apply an external magnetic field perpendicular to the plane of the bonded glass substrate. Through FEM modeling and experimental verification of the DMC microseparator, it was found that the DMC microseparator had characteristic limitations. For instance, using the geometries of the designed DMC microseparator, RBC's placed within $\sim 25 \mu \mathrm{m}$ of a square wire along the bottom of the microchannel, were not affected by the separation force, thereby decreasing the separation efficiency. While the PMC mode (Fig.1) is more difficult to fabricate than the DMC mode, the simulation analysis (Fig.2) shows that the PMC mode has definite advantages in this application including a stronger magnetic separation force generated around the square wire, which exponentially increases at a distance of $\sim 50 \mu \mathrm{m}$ from the wire edge toward the wire's edge along the bottom of microchannel. This paper describes the design and fabrication of the PMC microseparator (Fig.3), and reports on the quantitative performance of the PMC microseparator for separating WBC's from whole blood for genomic analysis and for removing a large percentage of the RBC's that act as inhibitors [3] for subsequent genomic analysis.

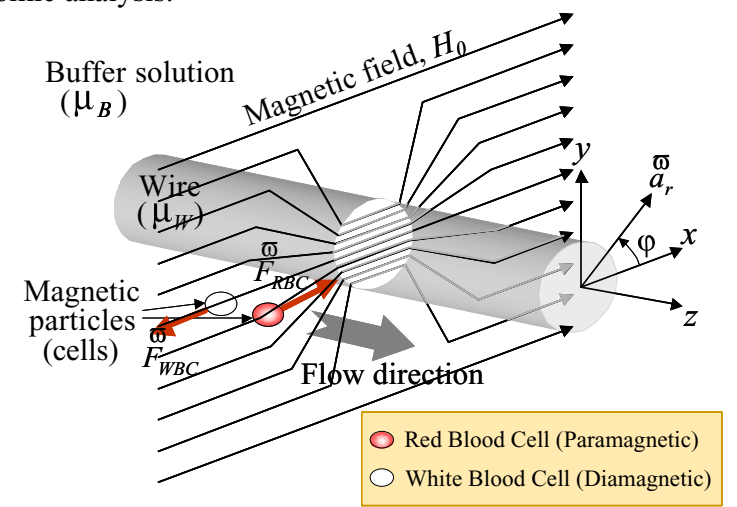

Figure 1. The working principle of the PMC microseparator.
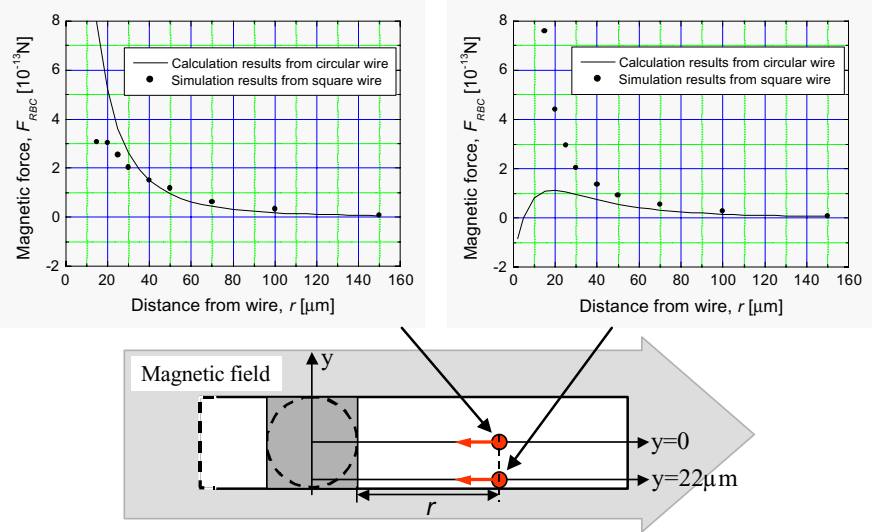

Figure 2. Calculation and simulation magnetic forces on $R B C$.

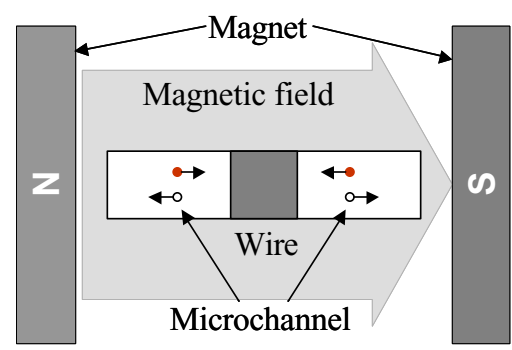

(a)

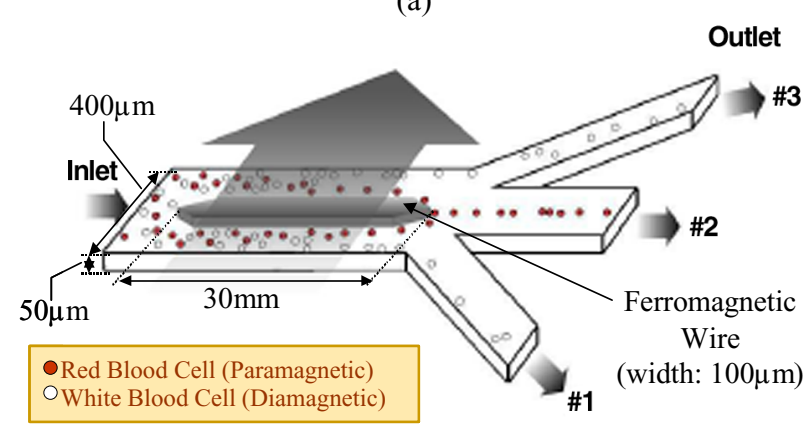

(b)

Figure 3. Illustrations of the PMC microseparator having one inlet and three outlets: (a) cross section view; (b) perspective view.

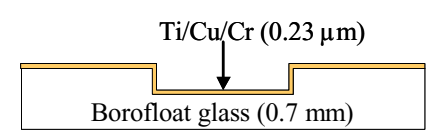

(a) Channel define $\&$ seed layer deposition

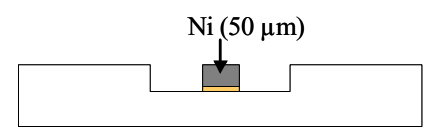

(b) Nickel electroplate

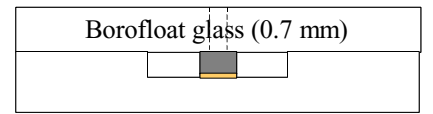

(c) Glass-to-glass bonding

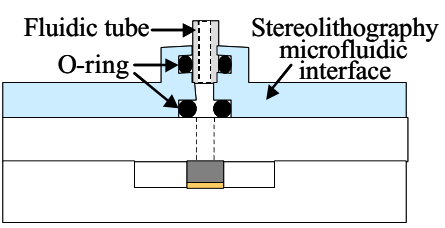

(d) Interface integration
Figure 4. Microfabrication process. 


\section{RESULTS AND CONCLUSION}

Figure 4 illustrates the microfabrication process of the PMC microseparator. Figure 5 shows the fabricated microseparator and instrumental setup implemented using a permanent magnet to create the external magnetic flux of $0.2 \mathrm{~T}$. Figures $6(\mathrm{a})$ and (b) show images of blood cells flowing at a velocity of $0.2 \mathrm{~mm} / \mathrm{s}$ through the microchannel with and without an external magnetic field applied. Figures 7(a) and (b) display images of blood cells flowing at velocities of $0.1 \mathrm{~mm} / \mathrm{sec}$ and $0.6 \mathrm{~mm} / \mathrm{s}$. The images show that RBC's are forced towards the wire with the application of an external magnetic field and accumulate at the wire edge. The relative percentage of RBCs at the outlets (Fig.8(a)), measured using a hemacytometer, shows that the PMC microseparator separates out $68.2 \%$ RBC's from whole blood at $0.1 \mathrm{~mm} / \mathrm{s}$ flow velocity, agreeing well with the estimated results from FEM modeling (Fig.8(b)). By monitoring WBC's dyed with a fluorescent probe (Fig.9), the relative percentage of WBC's at the outlets was measured as shown in Fig.10, and shows that WBC's are not influenced by a $0.2 \mathrm{~T}$ external magnetic flux applied to the PMC microseparator and separate away from the integrated wire due to Fickian diffusion.

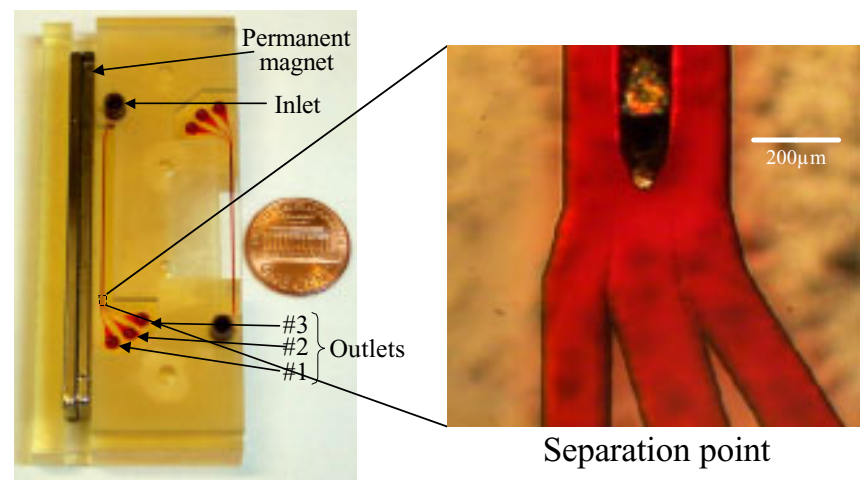

Figure 5. Fabricated microseparator and instrument setup.

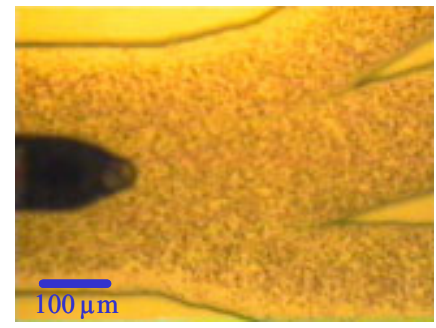

(a) Without magnetic field

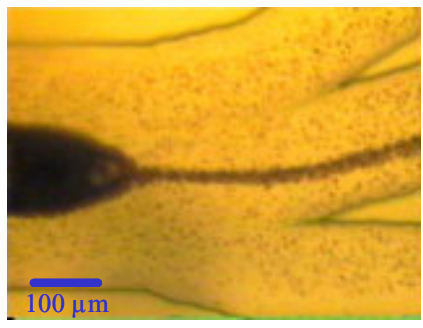

(b) With $0.2 \mathrm{~T}$ magnetic field
Figure 6. Blood cells passing through the microchannel of the PMC microseparator at a velocity of $0.2 \mathrm{~mm} / \mathrm{s}$.

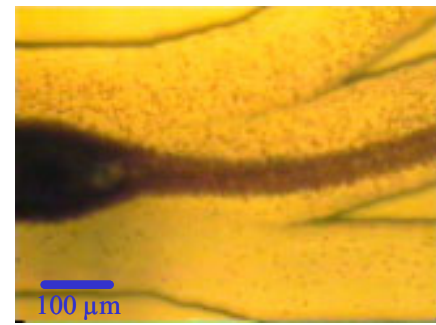

(a) $0.1 \mathrm{~mm} / \mathrm{s}$

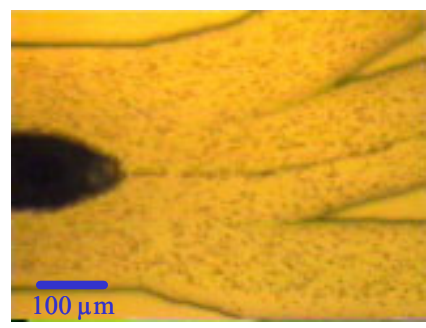

(b) $0.6 \mathrm{~mm} / \mathrm{s}$
Figure 7. Blood cells passing through the microchannel of the PMC microseparator at varying flow velocities with $0.2 T$ magnetic field applied.

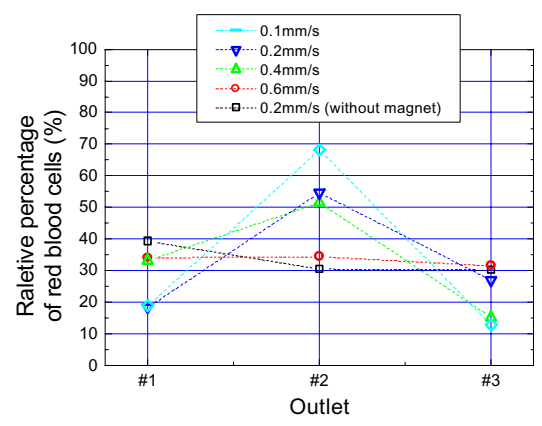

(a) Measured results

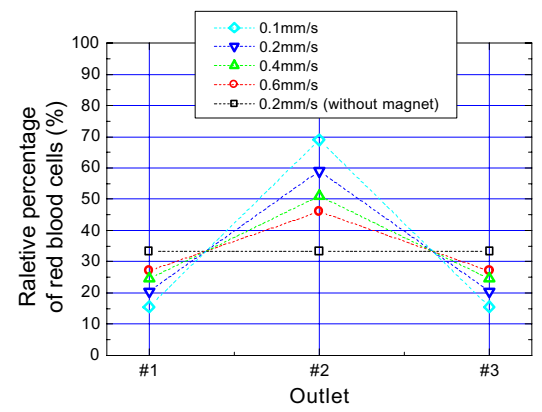

(b) Estimated results

Figure 8. The relative percentage of $R B C$ 's from the outlets of the PMC microseparator for varying flow velocity.

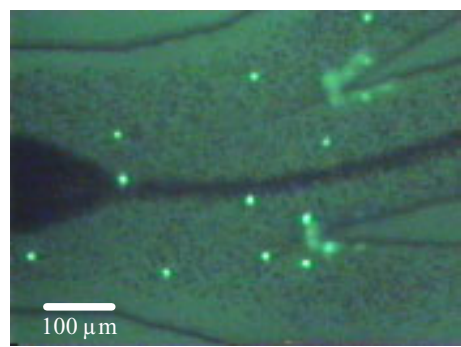

Figure 9. Fluorescent probed WBC's passing through the microchannel of the PMC microseparator at a velocity of $0.2 \mathrm{~mm} / \mathrm{s}$.

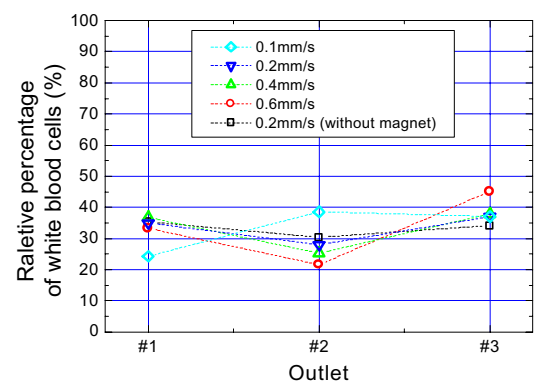

Figure 10. The relative percentage of WBC's from the outlets of the $P M C$ microseparator for varying flow velocity.

\section{ACKNOWLEDGEMENTS}

This work was supported by the National Institutes of Health (NIH) / National Institute for Environmental Health Sciences (NIEHS) under Grant ES10846.

\section{REFERENCES}

1. M. Franzreb, W. Holl, and C. Hoffmann, U.S. Patent No. 2002/0088741. 2. K.-H. Han, J. P. Landers, and A. B. Frazier, Transducers' 03, pp.1229-1232.

3. M. M. Hämäläinen, J. U. Eskola, J. Hellman, and K. Pulkki, Clinical Chemistry, 45, 465 (1999). 